$1-1-2004$

\title{
Resonant enhancement of two-photon absorption in substituted fluorene molecules
}

\author{
Joel M. Hales \\ University of Central Florida \\ David J. Hagan \\ University of Central Florida \\ Eric W. Van Stryland \\ University of Central Florida

\section{K. J. Schafer \\ University of Central Florida}

\section{A. R. Morales \\ University of Central Florida}

Find similar works at: https://stars.library.ucf.edu/facultybib2000

University of Central Florida Libraries http://library.ucf.edu

See next page for additional authors

This Article is brought to you for free and open access by the Faculty Bibliography at STARS. It has been accepted for inclusion in Faculty Bibliography 2000s by an authorized administrator of STARS. For more information, please contactSTARS@ucf.edu.

\section{Recommended Citation}

Hales, Joel M.; Hagan, David J.; Van Stryland, Eric W.; Schafer, K. J.; Morales, A. R.; Belfield, K. D.; Pacher, P.; Kwon, O.; and Bredas, J. L., "Resonant enhancement of two-photon absorption in substituted fluorene molecules" (2004). Faculty Bibliography 2000s. 4398.

https://stars.library.ucf.edu/facultybib2000/4398

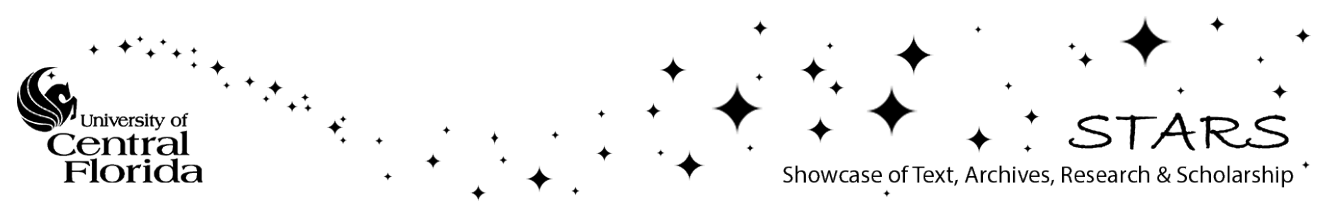




\section{Authors}

Joel M. Hales, David J. Hagan, Eric W. Van Stryland, K. J. Schafer, A. R. Morales, K. D. Belfield, P. Pacher, O. Kwon, and J. L. Bredas 


\section{Resonant enhancement of two-photon absorption in substituted fluorene molecules}

Cite as: J. Chem. Phys. 121, 3152 (2004); https://doi.org/10.1063/1.1770726

Submitted: 19 January 2004 . Accepted: 18 May 2004 . Published Online: 30 July 2004

Joel M. Hales, David J. Hagan, Eric W. Van Stryland, K. J. Schafer, A. R. Morales, K. D. Belfield, P. Pacher, O. Kwon, E. Zojer, and J. L. Bredas

\section{ARTICLES YOU MAY BE INTERESTED IN}

Non-degenerate two photon absorption enhancement for laser dyes by precise lock-in detection

AIP Advances 5, 127138 (2015); https://doi.org/10.1063/1.4939568

Effects of $\pi$ centers and symmetry on two-photon absorption cross sections of organic chromophores

The Journal of Chemical Physics 114, 9813 (2001); https://doi.org/10.1063/1.1371258

Two-photon absorption spectroscopy of stilbene and phenanthrene: Excited-state analysis and comparison with ethylene and toluene

The Journal of Chemical Physics 146, 174102 (2017); https://doi.org/10.1063/1.4982045

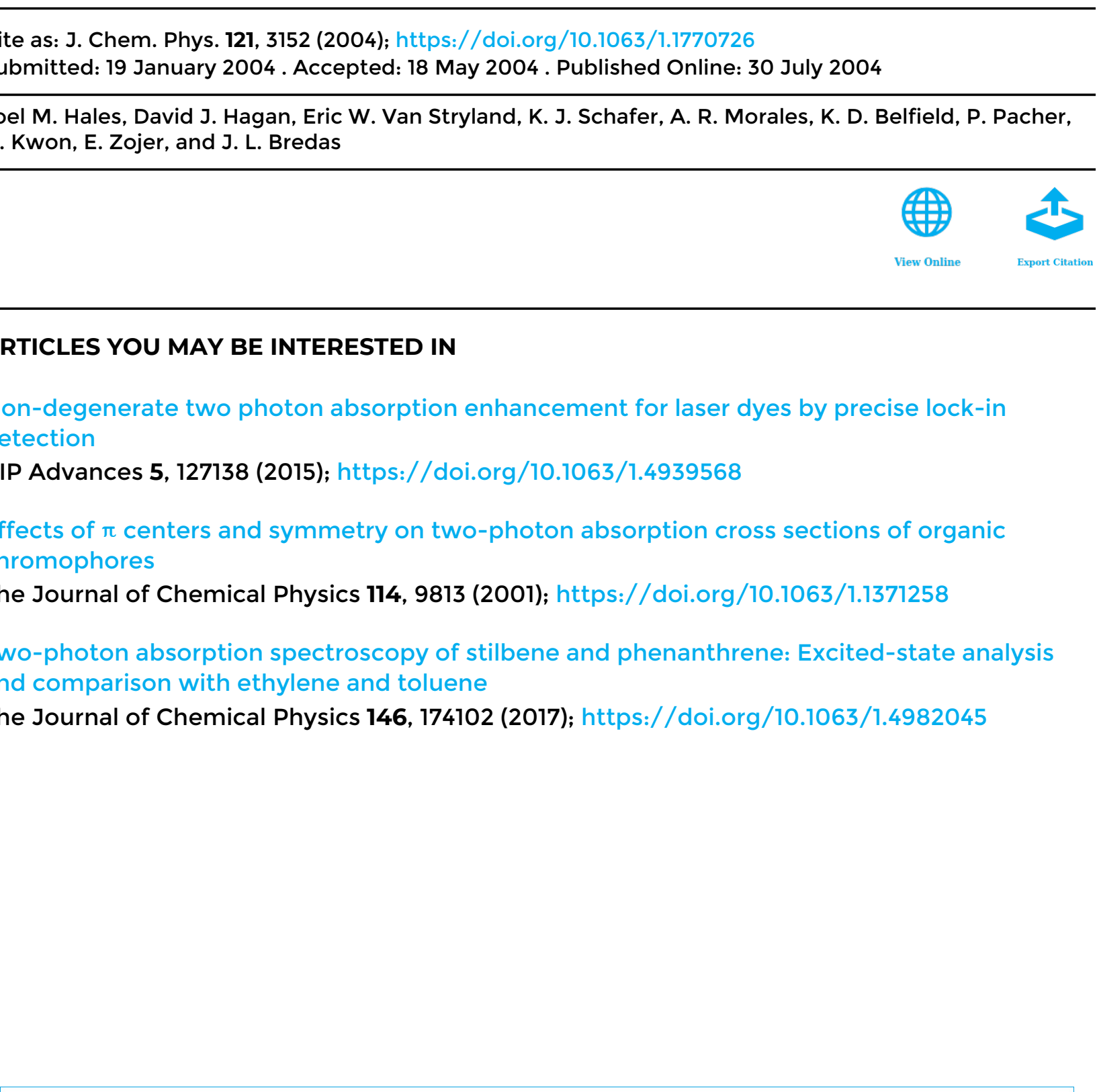

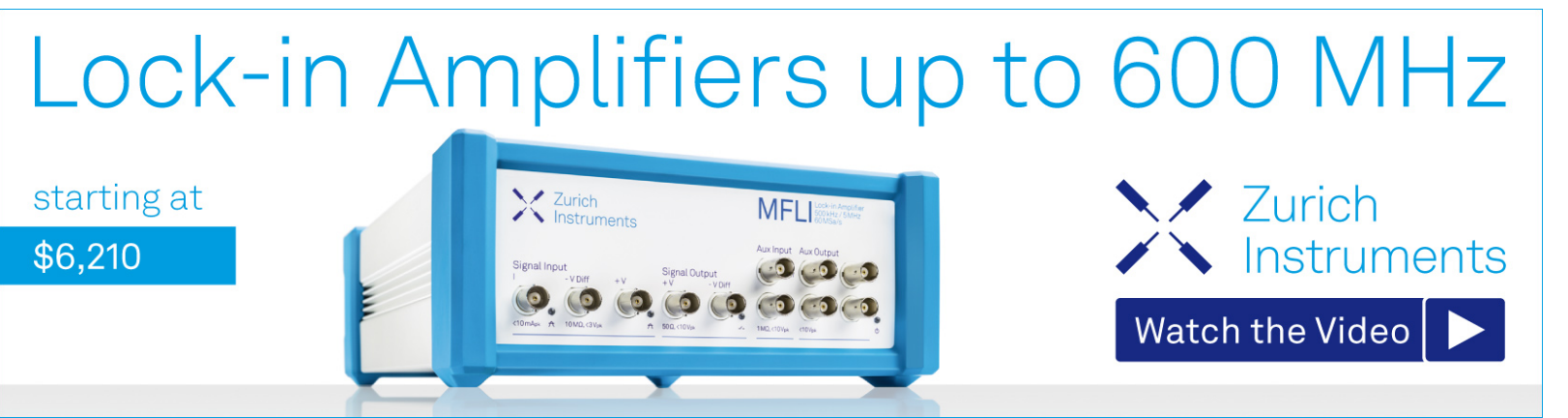

J. Chem. Phys. 121, 3152 (2004); https://doi.org/10.1063/1.1770726

121, 3152

(c) 2004 American Institute of Physics. 


\title{
Resonant enhancement of two-photon absorption in substituted fluorene molecules
}

\author{
Joel M. Hales, ${ }^{\text {a) }}$ David J. Hagan, and Eric W. Van Stryland \\ School of Optics/CREOL, University of Central Florida, Orlando, Florida 32816-2700 \\ K. J. Schafer, A. R. Morales, and K. D. Belfield \\ Department of Chemistry, University of Central Florida, Orlando, Florida 32816-2700 \\ P. Pacher, O. Kwon, E. Zojer, and J. L. Bredas \\ Department of Chemistry, The University of Arizona, Tucson, Arizona 85721-0041 and School of Chemistry \\ and Biochemistry, Georgia Institute of Technology, Atlanta, Georgia 30332-0400
}

(Received 19 January 2004; accepted 18 May 2004)

\begin{abstract}
The degenerate and nondegenerate two-photon absorption (2PA) spectra for a symmetric and an asymmetric fluorene derivative were experimentally measured in order to determine the effect of intermediate state resonance enhancement (ISRE) on the 2PA cross section $\delta$. The ability to tune the individual photon energies in the nondegenerate 2PA (ND-2PA) process afforded a quantitative study of the ISRE without modifying the chemical structure of the investigated chromophores. Both molecules exhibited resonant enhancement of the nonlinearity with the asymmetric compound showing as much as a twentyfold increase in $\delta$. Furthermore, the possibility of achieving over a one order of magnitude enhancement of the nonlinearity reveals the potential benefits of utilizing ND-2PA for certain applications. To model ISRE, we have used correlated quantum-chemical methods together with the perturbative sum-over-states (SOS) expression. We find strong qualitative and quantitative correlation between the experimental and theoretical results. Finally, using a simplified three-level model for the SOS expression, we provide intuitive insight into the process of ISRE for ND-2PA. 두 2004 American Institute of Physics. [DOI: 10.1063/1.1770726]
\end{abstract}

\section{INTRODUCTION}

Two-photon absorption (2PA) has attracted appreciable interest in the fields of photonics, chemistry, and biology due to the emergence of technologies that can exploit it. Among these are photodynamic cancer therapy, ${ }^{1}$ three-dimensional fluorescence imaging, ${ }^{2}$ microfabrication and optical data storage, ${ }^{3}$ as well as optical power limiting. ${ }^{4}$ The two key features of 2PA which make it an ideal candidate for the above applications are (1) its quadratic dependence on the incident irradiance which provides spatial selectivity in three dimensions and (2) the improved penetration depth into an absorbing medium afforded by the use of longer wavelength photons. Designing materials which exhibit large 2PA cross sections not only reduces the tolerances on devices employing 2PA by increasing their sensitivity but also allows one to reduce the intensity of the excitation source and therefore ameliorate the conditions with regard to optical damage. Organic molecules are promising candidates for efficient twophoton absorbers because their material properties can be tailored through molecular engineering. Consequently, design strategies for optimizing an organic compound's twophoton absorbing properties are crucial. Guidelines for such structure/property relations are becoming much more viable as studies combining quantum-chemical analysis and experimental data become more prevalent.

\footnotetext{
${ }^{a}$ Author to whom correspondence should be addressed; 4000 Central Florida Blvd., Orlando, FL 32816-2700; Fax: (407) 823-6880. Electronic mail: jhales@mail.ucf.edu
}

Several studies investigating these chemical structurenonlinear optical property relationships for organic materials have already proven successful. Optimization strategies have included extension of the chain length in $\pi$-conjugated systems, ${ }^{5}$ altering the strengths of the electron donating (D) and accepting (A) end groups in both quadrupolar ${ }^{6-8}$ and dipolar ${ }^{9}$ type compounds, as well as synthesizing compounds with multipolar geometries. ${ }^{10}$

Another far less investigated avenue for improving the 2PA properties of organics is resonance enhancement. When the energy difference between the photon energy and the energy of the nearest one-photon allowed state (the detuning energy) is reduced, dramatic enhancement of the 2PA will be observed. ${ }^{11}$ However, it should be noted that the minimum detuning energies achievable are inevitably limited by the natural linewidth of the transition (and any associated inhomogeneous broadening). Beyond this point, simultaneous 2PA, an instantaneous third-order nonlinear process, is dominated by sequential two-photon absorption (or excited-state absorption), an effective third-order nonlinearity, which results in the loss of any potential benefits true 2PA affords.

Of particular interest is the case where at least one of the incident photons approaches a resonance with the lowest one-photon allowed state, a condition known as intermediate state resonance enhancement (ISRE). The work devoted to exploiting this condition for the purpose of maximizing a molecule's 2PA properties has been promising and yet limited in its scope. Pati, Marks, and Ratner ${ }^{12}$ used conformational changes in a group of dipolar compounds to effec- 
tively alter the detuning energy to reach this resonance enhancement. Kogej et al. ${ }^{13}$ and Zojer et al. ${ }^{7}$ explored the possibilities of this enhancement in dipolar and quadrupolar systems, respectively, by altering the ground-state polarization, which in turn gave rise to a smaller detuning energy. Barzoukas and Blanchard-Desce ${ }^{14}$ also investigated resonance enhancement in both dipolar and quadrupolar systems as a function of the mixing of neutral and zwitterionic forms of the molecule. Each of the previous groups utilized quantum chemical analysis for their studies. In contrast, Drobizhev et al. ${ }^{15}$ and Kamada et al. ${ }^{16}$ showed experimental evidence of this enhancement process in substituted porphyrin systems and symmetric substituted diacetylenes, respectively. However, in all these cases the groups focused on degenerate 2PA (D-2PA), a process in which the sample simultaneously absorbs two photons of identical (or degenerate) energies. Since D-2PA into the two-photon allowed excited state $\left|e^{\prime}\right\rangle$ of the molecule was studied $\left(E_{g e^{\prime}} \approx 2 \hbar \omega\right)$, resonance enhancement was achieved if the energy of the molecule's lowest lying one-photon allowed state $|e\rangle$ approached half the energy of the two-photon state (i.e., $E_{g e}$ $\left.\rightarrow E_{g e^{\prime}} / 2\right)$. Therefore, in order to investigate the effect of this resonance condition, several molecules with slightly different structures (and hence slightly different energy levels) had to be studied. It is difficult to ascertain the value of enhancement of 2PA specifically due to resonance in these cases since altering a molecule's structure to satisfy the above condition will inevitably alter its other optical properties (e.g., state and transition dipole moments). If, instead of altering the position of the first excited state (i.e., $E_{g e} \rightarrow \hbar \omega$ for $\omega$ held constant), the photon energy is adjusted to approach resonance (i.e., $\hbar \omega \rightarrow E_{g e}$ for $E_{g e}$ held constant), the effect of resonance enhancement on $2 \mathrm{PA}$ can be studied directly.

In this paper, we report on the use of nondegenerate $2 \mathrm{PA}$ (ND-2PA) to vary the energies of the individual photons, $\hbar \omega_{1}$ and $\hbar \omega_{2}$, while keeping the two-photon energy, $\hbar\left(\omega_{1}\right.$ $\left.+\omega_{2}\right)$, constant. This allows us to vary the detuning from the intermediate state while accessing the same final states. In this way, we can quantitatively study the effect of ISRE in a single molecule. Through the direct investigation of ISRE afforded by this route we hope to provide greater insight into the nature of resonance enhancement. Furthermore, evidence of significant enhancement suggests promising applications using ND-2PA. In the following sections, we will address (1) the one-photon spectroscopic properties of the two fluorene derivatives used in this study, (2) the techniques utilized for degenerate and nondegenerate 2PA spectroscopy, (3) the 2PA spectra generated by these two techniques, and (4) the quantum-chemical calculations, which allowed us to further investigate the relationship between detuning energy and resonance enhancement. We note that Baltramiejunas et al. ${ }^{17}$ investigated the enhancement of ND-2PA due to deep local levels as intermediate states in $\mathrm{ZnO}$ and $\mathrm{ZnSe}$ semiconductor crystals.

\section{EXPERIMENTAL SECTION}

\section{A. Materials}

The chemical structures for the two organic molecules we have chosen to investigate are shown in Fig. 1. Com-
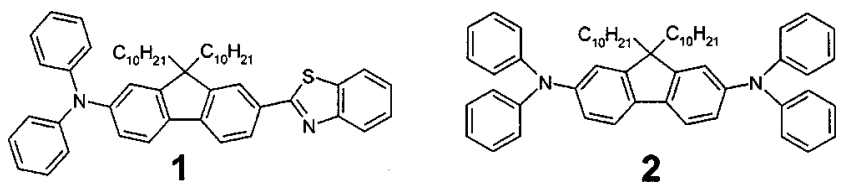

FIG. 1. Molecular structures of fluorene derivatives used in this study. 1, asymmetric $D-\pi$ - $A$ compound. 2, symmetric $D-\pi-D$ compound.

pound 1 has an asymmetric $D-\pi-A$ structure where $D$ refers to a diphenylamine electron donating moiety, $\pi$ refers to the conjugated aromatic fluorene core, and $A$ denotes a benzothiazole electron acceptor group. The second compound is a symmetric $D-\pi-D$ compound where the conjugated core lies between the two diphenylamine end groups. The details for the synthesis procedures for these compounds can be found in Ref. 18.

\section{B. Methods}

\section{Linear spectroscopy}

All spectroscopic measurements (both linear and nonlinear) were performed on solutions of the two compounds in hexane (spectroscopic grade from Aldrich). Hexane was chosen to minimize the effects of solvent polarity on the nonlinear properties of the samples. For the linear absorption and fluorescence measurements concentrations of approximately $10^{-6} \mathrm{M}$ were used. Absorption spectra were obtained using a Cary-3 UV-visible spectrophotometer. The fluorescence spectra were measured with a fully automated spectrofluorimeter (QM-6/2003, Photon Technology International) under $90^{\circ}$ excitation in a $T$-format method. Quantum yields $(\eta)$ of the compounds in solutions were measured using a standard method, ${ }^{19}$ relative to Rhodamine $6 \mathrm{G}$ in ethanol $(\eta=0.94) .{ }^{20}$ In addition, a more detailed analysis of the spectroscopic properties of the two compounds including the steady-state excitation anisotropy spectra which can determine energetic positions of excited states can be found in Ref. 21.

\section{Degenerate 2PA spectroscopy}

The method used for characterization of the degenerate 2PA spectra for these compounds was two-photon fluorescence (2PF) spectroscopy. Here, a strong tunable pump beam excites the material via 2PA and the total integrated fluorescence is monitored as a function of input frequency. The femtosecond source used for the pump beam is a Ti:sapphire based laser system (CPA-2001, CLARK-MXR) which provides laser pulses at $775 \mathrm{~nm}$ of $150 \mathrm{fs}$ duration at a $1 \mathrm{kHz}$ repetition rate. This laser, in turn, pumps an optical parametric amplifier (OPA) system (TOPAS, Light Conversion), which can be tuned from $570-2100 \mathrm{~nm}(0.6-2.2 \mathrm{eV})$ and provides up to $60 \mu \mathrm{J}$ of energy. This output energy is then attenuated and 10-150 nJ are used to produce the $2 \mathrm{PF}$. The sample solutions were contained within $1 \mathrm{~cm}$ path length quartz cuvettes and the concentrations used were approximately $10^{-4} \mathrm{M}$.

The sample is excited with a collimated beam over the full path length of the cell in such a manner as to minimize reabsorption of the emission. Following excitation of the 
sample, the full two-photon induced fluorescence spectrum is recorded to make certain that the up-converted fluorescence spectrum is independent of pump frequency (which is not always the case in some materials ${ }^{22}$ ). The $2 \mathrm{PF}$ signal was acquired for a number of different pump irradiances to ensure that it exhibits the proper quadratic dependence. The fluorescence measurement made is a relative one, ${ }^{8}$ implying that the $2 \mathrm{PF}$ results obtained for the sample under investigation are calibrated against well-know reference standards: fluorescein in water $(p \mathrm{H}=11)$ (Ref. 23) and 1,4-bis(2methylstyryl)benzene in cyclohexane. ${ }^{24}$

\section{Nondegenerate 2PA spectroscopy}

For nondegenerate 2PA spectroscopy we make use of a femtosecond white-light continuum (WLC) pump-probe nonlinear spectrometer. In this section we provide a brief explanation of the technique used and refer the reader to a more detailed description of the method provided in Ref. 25. In this experimental setup, the OPA (the same one used for the $2 \mathrm{PF}$ method) provides a strong pump beam whose irradiance can be varied from 30 to $90 \mathrm{GW} / \mathrm{cm}^{2}$. Another identical OPA is used to generate a weak, broadband WLC probe beam by focusing 1-2 $\mu \mathrm{J}$ of $1300 \mathrm{~nm}$ light into a $2.5 \mathrm{~mm}$ thick piece of calcium fluoride. The ND-2PA process requires simultaneous absorption of one photon from both the pump and probe beams. Since the WLC possesses a broad bandwidth (400-700 nm or $0.7-3.1 \mathrm{eV})$, the full 2PA spectrum of the compound can be sampled provided the proper pump wavelength is chosen. In fact, the broadband nature of the WLC probe should, in principle, allow for a method which is single-shot in nature. However, in practice, the creation of the WLC imposes chirp on the probe beam which requires us to vary the temporal delay of the pump with respect to the probe in order to obtain the entire 2PA spectrum. Furthermore, the nondegenerate nature of the experiment introduces temporal walkoff effects where the difference in group velocities between the pump and probe pulses can cause a reduction in the true nonlinear absorption signal. By correcting for this temporal chirp and accounting for the linear propagation effects, we can effectively characterize a sample's ND-2PA spectrum.

We would like to note that in these experiments we have chosen to keep the energy of the pump photon less than half the energy of the linear absorption edge of the sample under investigation. In doing this, we assure negligible D-2PA of the pump beam. This allowed us to avoid absorption of the pump beam and subsequent excited state absorption of the WLC probe beam which could have interfered with our data analysis. $^{25}$

The sample solutions used were placed within $1 \mathrm{~mm}$ path length glass cuvettes and the sample concentrations were approximately $10^{-2} \mathrm{M}$. In order to confirm that aggregation-type effects (e.g., dimer formation) did not interfere with our measurements, we carried out the D-2PA measurements with these highly concentrated solutions as well. The discrepancies we noticed between the 2PF spectra taken with the lower concentration samples $\left(10^{-4} \mathrm{M}\right)$ and the more highly concentrated ones were minor, thus confirming that aggregation effects were negligible. This is due to the pres-
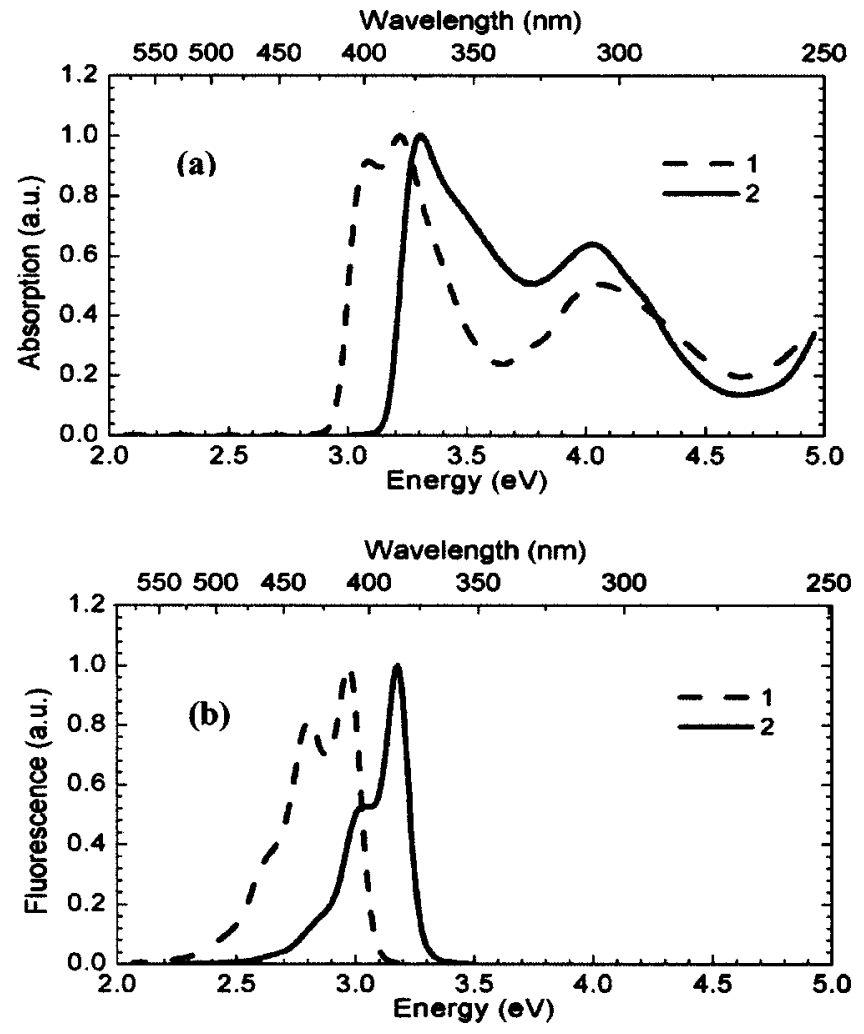

FIG. 2. Absorption (a) and fluorescence (b) spectra of compounds 1 (dashed) and 2 (solid) in hexane.

ence of the appended alkane chains on the investigated molecules which afford significant solubility. Furthermore, it should be noted that the linear absorption at the pump and probe wavelengths in the nondegenerate WLC measurements described below were negligible.

\section{Theoretical methodology}

The molecular ground-state geometries were optimized using the semiempirical AM1 Hamiltonian. ${ }^{26}$ All calculations were performed on isolated molecules, thus neglecting solvent effects. This approximation seems reasonable because hexane was chosen as the solvent in the experiments for the specific reason of minimizing solvent effects. The excitedstate energies, state- and transition-dipole moments were obtained by performing a highly correlated calculation where the INDO (Ref. 27) Hamiltonian is coupled to a multireference determinant single and double configuration interaction (MRD-CI) technique (Refs. 28 and 29) using the MatagaNishimoto potential ${ }^{30}$ to express the Coulomb repulsion term. From the excited state energies and transition dipoles we then calculate the degenerate and nondegenerate 2PA cross sections as will be detailed below.

\section{RESULTS AND DISCUSSION}

\section{A. One-photon spectroscopy}

The one-photon absorption and fluorescence spectra for compounds 1 and 2 in hexane are shown in Fig. 2. The linear absorption spectra for both compounds have well-defined peaks identifying the first two strongly allowed one-photon 

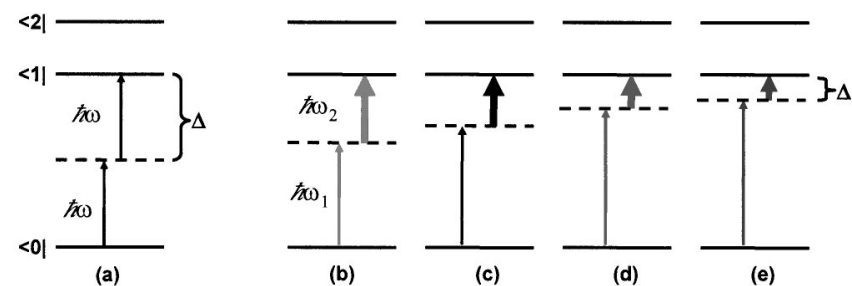

FIG. 3. Schematic representation of experiments performed for 2PA into the first excited state of compound 1. (a) Shows the D-2PA measurement (2PF) whereas (b)-(e) show ND-2PA (WLC); in the latter cases, $\hbar \omega_{1}$ represents the probe photons and $\hbar \omega_{2}$ the pump photons. The exact photon energies for each experiment are given in Table I.

transitions. Furthermore, both compounds exhibit vibronic structures in the absorption and fluorescence spectra which have a progression of approximately $1300 \mathrm{~cm}^{-1}$ corresponding to $\mathrm{C}-\mathrm{C}$ stretching vibrations. The quantum yields for compounds 1 and 2 in hexane were determined to be 0.7 and 0.4 , respectively.

\section{B. Two-photon spectroscopy}

Schematic representations of the 2PA spectroscopy experiments carried out on compounds 1 and 2 are shown in Figs. 3 and 4, respectively. Figure 3 illustrates 2PA into the first excited state of the $D-\pi-A$ molecule whereas Fig. 4 denotes 2PA into the first two-photon allowed state of the $D-\pi$ - $D$ molecule. As compound 2 is close to a centrosymmetric system, 2PA into its strongly one-photon allowed lowest excited singlet state is negligible; consequently, nonlinear absorption into its first two-photon allowed state was studied. For the asymmetric molecule (compound 1), 2PA is symmetry allowed into every excited state. This permitted us to investigate 2PA also into the lowest excited state.

Figures 3(a) and 4(a) schematically describe the D-2PA experiments, which were carried out by the $2 \mathrm{PF}$ method. The remaining figures [Figs. 3(b)-3(e) and 4(b)-4(d)] denote ND-2PA, as studied in the WLC pump-probe experiment. The arrows denoting the pump photon are shown in bold since the pump beam is the high-intensity beam. $\Delta$ represents the detuning energy between the first excited state and the energy of the high-energy photon (the probe photon in ND2PA).

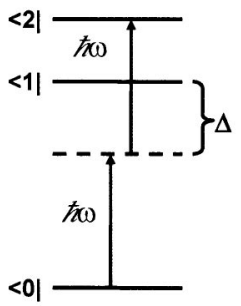

(a)

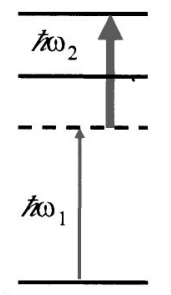

(b)

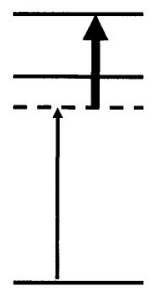

(c)

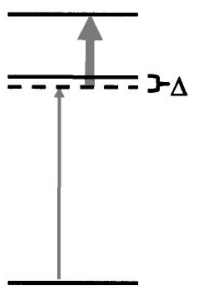

(d)
FIG. 4. Schematic representation of experiments performed for 2PA into the first two-photon allowed state of compound 2. (a) Shows the D-2PA measurement (2PF) whereas (b)-(d) show ND-2PA (WLC); in the latter cases, $\hbar \omega_{1}$ represents the probe photons and $\hbar \omega_{2}$ the pump photons. The photon energies for each experiment are given in Table II.

The values of the photon energies are given in Tables I and II. Although the probe is actually composed of a broad continuum of energies, for the sake of simplicity it is represented here as the energy necessary to reach the peak of the 2PA spectrum. The sum of the energies of the incident photons is resonant with the final state which can be verified in Tables I and II, considering that the experimentally determined state energies are $E_{2 \mathrm{PA}}=3.22 \mathrm{eV}$ for compound 1 and $E_{2 \mathrm{PA}} \approx 4.00 \mathrm{eV}$ for compound 2 .

The two-photon absorption spectra, as acquired from the $2 \mathrm{PF}$ experiment (i.e., D-2PA) and the WLC pump-probe experiment ${ }^{31,32}$ for compounds 1 and 2, are shown in Figs. 5(a) and 6(a), respectively. The corresponding pump energies for the WLC measurements are listed in the inset. The solid lines are sums of two Gaussian functions of width $0.3 \mathrm{eV}$ which are fitted to the experimental data. Furthermore, the centers of the Gaussian functions are kept constant for the curves in each individual figure. The one exception is curve (d) in Fig. 6(a). The peak of the nonlinear absorption spectrum is slightly blue shifted $(\sim 0.05 \mathrm{eV})$ with respect to the other three curves; this is within the bounds determined by the experimental errors.

First, from Fig. 5(a) we note that the peak positions of the 2PA spectra correlate quite well with the position of the first excited state for compound 1 illustrated by the location of the peak of the linear absorption spectrum shown in Fig. 2(a) $\left(E_{01}=3.22 \mathrm{eV}\right)$. 2PA into higher lying excited states is also evident; however, the contours of the 2PA spectra do not

TABLE I. 2PA spectral data for compound 1. The photon energies $\left(\hbar \omega_{1}, \hbar \omega_{2}\right.$ in eV) for the experiments schematically represented in Fig. 3 are given. For the nondegenerate case, index 1 represents the probe photons and 2 the pump photons. The slightly different values for the probe energies in the calculations vs the experiments are due to the slight overestimation of the $S_{1}$ energy in the theoretical studies. Also given are the peak 2PA cross sections in GM units $\left(1 \times 10^{-50} \mathrm{~cm}^{4} \mathrm{sec}\right.$ photon ${ }^{-1}$ molecule $\left.{ }^{-1}\right)$ of the experimental $\left(\delta_{\text {exp }}\right)$ and sumover-states calculated ( $\left.\delta_{\text {Sos }}\right)$ 2PA spectra given in Fig. 5 as well as their associated resonance enhancements (ISRE $_{\text {exp }}, I_{S R E}$ SOS). The absolute errors for $\delta_{\text {exp }}$ are $\pm 15 \%$ and the relative errors from one wavelength to the next are $\pm 6 \%$.

\begin{tabular}{|c|c|c|c|c|c|c|c|c|}
\hline & \multicolumn{4}{|c|}{ Experimental } & \multicolumn{4}{|c|}{ Sum over states } \\
\hline & $\hbar \omega_{1}$ & $\hbar \omega_{2}$ & $\delta_{\exp }^{\max }$ & $\mathrm{ISRE}_{\text {exp }}$ & $\hbar \omega_{1}$ & $\hbar \omega_{2}$ & $\delta_{\mathrm{SOS}}^{\max }$ & ISRE $_{\text {SOS }}$ \\
\hline (a) & 1.61 & 1.61 & 72 & 1.00 & 1.72 & 1.72 & 65 & 1.00 \\
\hline (b) & 1.84 & 1.38 & 133 & 1.85 & 2.06 & 1.38 & 84 & 1.29 \\
\hline (c) & 2.19 & 1.03 & 168 & 2.33 & 2.41 & 1.03 & 124 & 1.91 \\
\hline (d) & 2.39 & 0.83 & 190 & 2.64 & 2.61 & 0.83 & 169 & 2.60 \\
\hline (e) & 2.53 & 0.69 & 239 & 3.32 & 2.75 & 0.69 & 216 & 3.32 \\
\hline
\end{tabular}


TABLE II. 2PA spectral data for compound 2. The photon energies $\left(\hbar \omega_{1}, \hbar \omega_{2}\right.$ in eV) for the experiments schematically represented in Fig. 4 are given. For the nondegenerate case, index 1 represents the probe photons and 2 the pump photons. Also given are the peak 2PA cross sections in GM units (1 $\times 10^{-50} \mathrm{~cm}^{4}$ sec photon ${ }^{-1}$ molecule $\left.{ }^{-1}\right)$ of the experimental $\left(\delta_{\text {exp }}\right)$ and sum-over-states calculated $\left(\delta_{\text {SOS }}\right) 2 \mathrm{PA}$ spectra given in Fig. 6 as well as their associated resonance enhancements (ISRE $\mathrm{exp}_{\text {, }}, \mathrm{ISRE}_{\mathrm{SOS}}$ ). The absolute errors for $\delta_{\exp }$ are $\pm 15 \%$ and the relative errors from one wavelength to the next are $\pm 6 \%$.

\begin{tabular}{|c|c|c|c|c|c|c|c|c|}
\hline & \multicolumn{4}{|c|}{ Experimental } & \multicolumn{4}{|c|}{ Sum over states } \\
\hline & $\hbar \omega_{1}$ & $\hbar \omega_{2}$ & $\delta_{\exp }^{\max }$ & $\mathrm{ISRE}_{\text {exp }}$ & $\hbar \omega_{1}$ & $\hbar \omega_{2}$ & $\delta_{\mathrm{SOS}}^{\max }$ & ISRE $_{\text {SOS }}$ \\
\hline (a) & 2.00 & 2.00 & 89 & 1.00 & 2.03 & 2.03 & 100 & 1.00 \\
\hline (b) & 2.35 & 1.65 & 169 & 1.90 & 2.41 & 1.65 & 132 & 1.32 \\
\hline (c) & 2.62 & 1.38 & 253 & 2.84 & 2.68 & 1.38 & 185 & 1.85 \\
\hline (d) & 3.02 & 1.03 & 427 & 4.80 & 3.03 & 1.03 & 391 & 3.91 \\
\hline
\end{tabular}

follow that of the linear spectrum. For the second compound, Fig. 6(a) shows negligible 2PA into the first excited state as expected for a system close to being centrosymmetric. The peak positions of the 2PA spectra denote the position of the first two-photon allowed state, $E_{2 \mathrm{PA}}=4.00 \mathrm{eV}$. The close proximity between this two-photon allowed state and the second one-photon allowed state shown in Fig. 2(b) $\left(E_{02}\right.$ $=4.03 \mathrm{eV}$ ) was verified by the quantum-chemical calculations which show that the two states are separated by less than $0.05 \mathrm{eV}$.

Second, Figs. 5(a) and 6(a) illustrate directly the effect of intermediate state resonance enhancement. As noted in Figs. 3 and 4, a decrease in the pump photon energy causes a reduction in the detuning energy between the probe photon energy and the first excited state (i.e., the intermediate state). This decreased detuning energy leads to resonant enhancement of the 2PA spectra, as evidenced in Figs. 5(a) and 6(a). In order to determine the magnitude of this resonance enhancement, each peak cross section (given in Tables I and II) was normalized to the value acquired from the D-2PA measurement because the degenerate measurement represents the largest possible detuning energy. The values of the ISRE are shown in Tables I and II. For the two-photon resonance, the data show over a threefold increase in $\delta$ for compound 1 and nearly a fivefold increase for compound 2 . It is important to realize here that while enhancement into higher-lying states (as is the case for 2) has been observed in molecules when using D-2PA (see above), enhancement into the first excited singlet state (as in 1) is a direct consequence of nondegenerate excitation. This phenomenon of enhanced two-photon transitions in molecules with permanent dipole moments was predicted theoretically by Scharf and Band ${ }^{33}$ in 1988. In addition, we would also like to note that 2PA into higher-level excited states of molecule 1 (shown for $E_{\exp }>3.22 \mathrm{eV}$ ) also exhibits resonance enhancement. For a pump photon energy of $0.83 \mathrm{eV}$ [indicated by curve $\mathrm{d}$ in Fig. 5(a)], the nondegenerate data show more than 20 times enhancement relative to the degenerate data at a value of $3.75 \mathrm{eV}$ for the sum of the two-photon energies. In this case, the probe photon energy is approximately $2.9 \mathrm{eV}$; based on the linear absorption spectrum for compound 1 (Fig. 2), this corresponds to a detuning energy of only $0.1 \mathrm{eV}$ relative to the first vibronic feature of the $S_{0} \rightarrow S_{1}$ excitation. Vibronic contributions can have a significant impact on resonant nonlinear optical responses. ${ }^{34,35}$ In this case, this vibronic feature determines the position of the intermediate state which affects resonance enhancement. Linear absorption of the WLC probe beam prevents the measurement of the ND-2PA spectrum for an even higher sum of photon energies. This is reflected in the truncation of the ND-2PA spectra at higher values of $E_{\exp }$ in the figures.
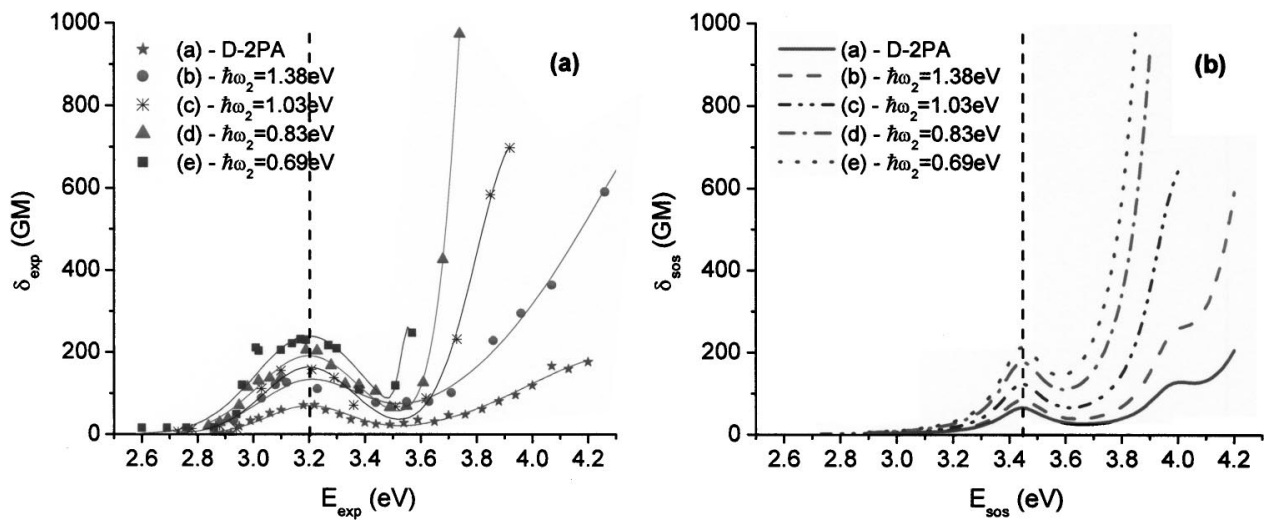

FIG. 5. 2PA spectra of compound 1, (a) shows the 2PA spectra for the experiments schematically represented in Fig. 3. The absolute errors for $\delta_{\text {exp }}$ are $\pm 15 \%$ and the relative errors from one wavelength to the next are $\pm 6 \%$. The symbols represent the $2 \mathrm{PA}$ cross sections acquired from the $2 \mathrm{PF}$ experiment and the WLC pump-probe experiment and the solid lines are fitting functions. (b) Shows the sum-over-states calculated 2PA spectra. The $y$ axes denote 2PA cross sections in GM units $\left(1 \times 10^{-50} \mathrm{~cm}^{4} \mathrm{sec}\right.$ photon ${ }^{-1}$ molecule $\left.{ }^{-1}\right)$ and the $x$ axes represent the sum of the two photon energies in $\mathrm{eV}$. The vertical dashed lines in both figures represent the position of the peak $2 \mathrm{PA}$ cross sections which are given in Table I. 

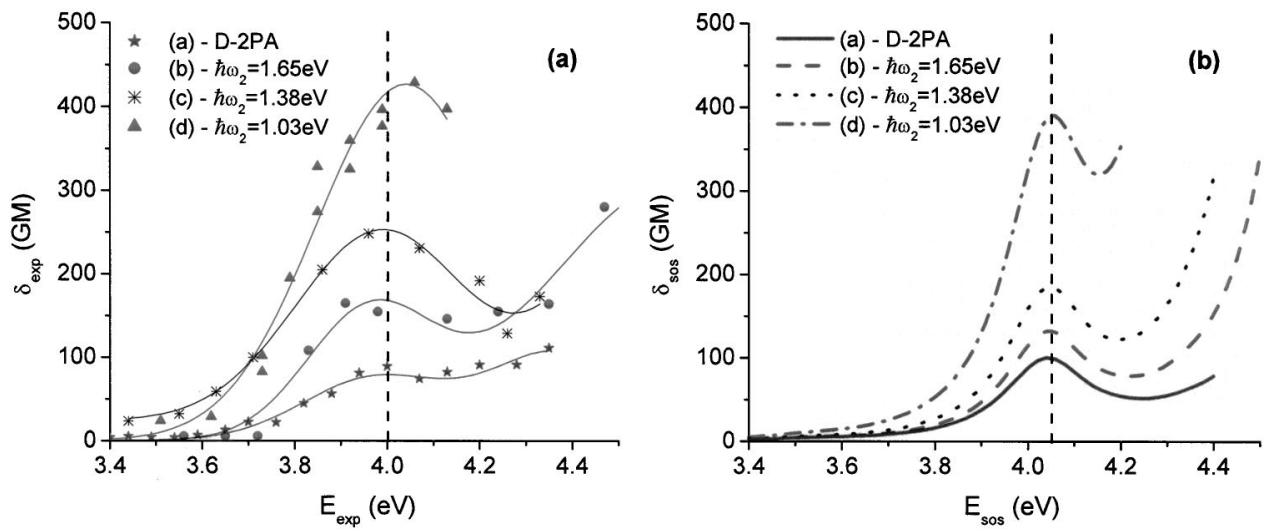

FIG. 6. 2PA spectra of compound 2. (a) Shows the 2PA spectra for the experiments schematically represented in Fig. 4. The absolute errors for $\delta_{\text {exp }}$ are $\pm 15 \%$ and the relative errors from one wavelength to the next are $\pm 6 \%$. The symbols represent the $2 \mathrm{PA}$ cross sections acquired from the $2 \mathrm{PF}$ experiment and the WLC pump-probe experiment and the solid lines are fitting functions. (b) Shows the sum-over-states calculated 2PA spectra. The $y$ axes denote 2PA cross sections in GM units $\left(1 \times 10^{-50} \mathrm{~cm}^{4} \mathrm{sec}\right.$ photon ${ }^{-1}$ molecule $\left.{ }^{-1}\right)$ and the $x$ axes represent the sum of the two photon energies in eV. The vertical dashed lines in both figures represent the position of the peak 2PA cross sections which are given in Table II.

\section{Quantum chemical analysis}

To gain a deeper understanding of the resonance enhancement processes, we have performed quantum-chemical calculations of ND-2PA relying on the perturbative sumover-states (SOS) approach given by Orr and Ward for the third-order molecular polarizability $\gamma\left(-\omega_{1} ; \omega_{1},-\omega_{2}, \omega_{2}\right)$ (Ref. 36) (here, contributions from the 300 lowest-lying excited states are considered in the perturbative expansion and the damping factor $\Gamma$ was taken to be $0.1 \mathrm{eV}$ for all transitions in accordance with previous publications ${ }^{8,13}$ ). The ND2PA cross section $\delta_{\mathrm{ND}}$ is then related to the imaginary part of the third-order polarizability $\gamma$ by ${ }^{37-39}$

$$
\delta_{\mathrm{ND}}=L_{1}^{2} L_{2}^{2} \frac{3 \hbar \omega_{1}^{2} \omega_{2} \operatorname{Im}\left[\gamma\left(-\omega_{1} ; \omega_{1},-\omega_{2}, \omega_{2}\right)\right]}{\left(\omega_{1}+\omega_{2}\right) \epsilon_{0} c^{2} n_{1} n_{2}},
$$

where index 1 refers to the probe beam and index 2 to the pump beam. $L$ is the local field factor and $n$ the refractive index of the medium. These were both set to 1 for the study of isolated molecules in vacuum. Equation (1) reduces to the well-known expression for the degenerate case (see, for example, Ref. 7 or 13) for $L_{1}=L_{2}, n_{1}=n_{2}$, and $\omega_{1}=\omega_{2}$. To account for the fact that the experimentally investigated sample is isotropic, the orientational average of $\gamma$ is used in Eq. (1).

The resulting degenerate and nondegenerate 2PA spectra for molecules 1 and 2 for the two compounds are given in Figs. 5(b) and 6(b). The pump energies were identical to those used in the experiments described above and are listed in Tables I and II.

For both molecules, the calculated SOS spectra describe very well the effect of resonance enhancement. Furthermore, the magnitudes of the degenerate and nondegenerate 2PA cross sections (both for the lowest two-photon allowed state and higher-lying states) agree well with the experimental data. As a result, the values for the intermediate-state resonance enhancement are consistent with the experimental results.

We note that for molecule 1 the spectral position of the calculated 2PA maxima is shifted with respect to experiment:
$E_{2 \mathrm{PA}}^{\prime}=3.44 \mathrm{eV}$ versus $E_{2 \mathrm{PA}}=3.22 \mathrm{eV}$ (where the prime denotes the theoretical value). This is a result of a slight overestimation of the position of the first excited state (by 0.22 $\mathrm{eV})$ in the quantum-chemical calculations; a similar discrepancy occurs for molecule $2\left(E_{01}^{\prime}=3.53 \mathrm{eV}\right.$ versus $E_{01}$ $=3.3 \mathrm{eV})$. The difference in $E_{01}$ values between the theoretical and experimental results manifests itself when plotting ISRE versus the detuning energy; however, the overall correlation between the experimental and SOS-generated 2PA spectra is quite satisfactory.

\section{Essential-state models}

Given that the full sum-over-states expression for $\gamma$ (including contributions from the first 300 excited states) provides an effective description of the ISRE phenomenon, it is useful to try and find approximations to the full SOS treatment, that would allow for a more simple picture and a greater insight into the nature of ISRE. In this context, Dirk, Cheng, and Kuzyk ${ }^{40}$ and Birge and Pierce ${ }^{41}$ have developed a three-level model for $\gamma$ while Mazumdar et al. ${ }^{42}$ investigated the roles of essential states in the third-order nonlinearity. In such approximate expressions the full SOS formula is truncated by assuming that there is a single excited state $|e\rangle$ that is strongly one-photon allowed and acts as an intermediate state for 2PA into the two-photon allowed states $\left|e^{\prime}\right\rangle$. When considering only resonant terms in D-2PA, the full SOS expression reduces to three terms: a dipolar term $(D)$, a two-photon term $(T)$, and a negative term $(N)$. The $D$ and $T$ terms contain two-photon resonances with $|e\rangle$ and

TABLE III. Selected INDO/MRD-CI calculated dipole moments (in Debye) and transition energies (in eV) for molecules 1 and 2.

\begin{tabular}{lrll}
\hline \hline \multicolumn{2}{c}{ Molecule 1 } & \multicolumn{2}{c}{ Molecule 2 } \\
\hline$E_{g e}$ & 3.44 & $E_{g e}$ & 3.53 \\
$\mu_{g e}$ & 10.31 & $\mu_{g e}$ & 8.78 \\
$\Delta \mu_{g e}$ & 7.78 & $E_{g e^{\prime}}$ & 4.05 \\
& & $\mu_{e e^{\prime}}$ & 5.80 \\
\hline \hline
\end{tabular}


$\left|e^{\prime}\right\rangle$, respectively. The $N$ term is related to one-photon resonances and therefore will not be discussed here. This simple three-state model for D-2PA has been successfully applied in numerous studies to date (see, for example, Ref. 7, 8, or 13).

Following the same approach, the $D$ and $T$ terms can also be derived for the nondegenerate case and can be written as

$$
\begin{aligned}
& \operatorname{Im} \gamma\left(-\omega_{1} ; \omega_{1},-\omega_{2}, \omega_{2}\right) \\
& \quad \approx \operatorname{Im}\left[\begin{array}{c}
\frac{\mu_{g e}^{2} \Delta \mu_{g e}^{2}}{\left(\Omega_{g e}-\hbar \omega_{1}-\hbar \omega_{2}\right)}\left\{\frac{1}{\left(\Omega_{g e}-\hbar \omega_{2}\right)\left(\Omega_{g e}-\hbar \omega_{1}\right)}+\frac{1}{\left(\Omega_{g e}-\hbar \omega_{2}\right)\left(\Omega_{g e}-\hbar \omega_{2}\right)}+\frac{1}{\left(\Omega_{g e}^{*}-\hbar \omega_{1}\right)\left(\Omega_{g e}-\hbar \omega_{1}\right)}+\frac{1}{\left(\Omega_{g e}^{*}-\hbar \omega_{1}\right)\left(\Omega_{g e}-\hbar \omega_{2}\right)}\right\} \\
+\sum_{e^{\prime}} \frac{\mu_{g e}^{2} \mu_{e e^{\prime}}^{2}}{\left(\Omega_{g e^{\prime}}-\hbar \omega_{1}-\hbar \omega_{2}\right)}\left\{\frac{1}{\left(\Omega_{g e}-\hbar \omega_{2}\right)\left(\Omega_{g e}-\hbar \omega_{1}\right)}+\frac{1}{\left(\Omega_{g e}-\hbar \omega_{2}\right)\left(\Omega_{g e}-\hbar \omega_{2}\right)}+\frac{1}{\left(\Omega_{g e}^{*}-\hbar \omega_{1}\right)\left(\Omega_{g e}-\hbar \omega_{1}\right)}+\frac{1}{\left(\Omega_{g e}^{*}-\hbar \omega_{1}\right)\left(\Omega_{g e}-\hbar \omega_{2}\right)}\right\}
\end{array}\right],
\end{aligned}
$$

where $\mu_{g e}$ is the transition dipole moment between the ground state $|g\rangle$ and $|e\rangle, \mu_{e e^{\prime}}$ is the transition moment between $|e\rangle$ and $\left|e^{\prime}\right\rangle$, and $\Delta \mu_{g e}$ is the difference between the permanent dipole moments in states $|g\rangle$ and $|e\rangle . \Omega_{g e}=E_{g e}$ $-i \Gamma_{g e}$ and $\Omega_{g e^{\prime}}=E_{g e^{\prime}}-i \Gamma_{g e^{\prime}}$, where $E_{g e}$ and $E_{g e^{\prime}}$ are the transition energies from the ground state to the relevant excited states and $\Gamma$ is the damping term associated with these states (set to a constant value of $0.1 \mathrm{eV}$, as mentioned above). It should be noted that Eq. (2) reproduces the terms applicable for D-2PA when $\hbar \omega_{1}=\hbar \omega_{2}=\hbar \omega$.

For the case of a resonance into a particular excited state ( $\hbar \omega_{1}+\hbar \omega_{2}=E_{g e}$ in the case of 2PA into the one-photon allowed state $|e\rangle$ and $\hbar \omega_{1}+\hbar \omega_{2}=E_{g e^{\prime}}$ for excitation into $\left|e^{\prime}\right\rangle$ ) and when the damping is much smaller than the photon energies, one obtains ${ }^{43}$ from Eqs. (2) and (1),

$$
\delta_{D,|e\rangle} \approx K \frac{\mu_{g e}^{2} \Delta \mu_{g e}^{2}}{\Gamma_{g e}}\left(\frac{1}{\hbar \omega_{1}}+\frac{1}{\hbar \omega_{2}}\right)^{2}
$$

for the $D$ term describing ND-2PA into $|e\rangle$ and

$$
\delta_{T,\left|e^{\prime}\right\rangle} \approx K \frac{\mu_{g e}^{2} \mu_{e e^{\prime}}^{2}}{\Gamma_{g e}}\left(\frac{1}{E_{e g}-\hbar \omega_{1}}+\frac{1}{E_{e g}-\hbar \omega_{2}}\right)^{2}
$$

for the $T$-term describing ND-2PA into $\left|e^{\prime}\right\rangle . K$ is a common factor [see also Eq. (1)] and is given by ${ }^{44}$

$$
K=\frac{3 L_{1}^{2} L_{2}^{2}}{5 n_{1} n_{2} c^{2} \epsilon_{0} \hbar} \frac{\hbar \omega_{2}\left(\hbar \omega_{1}\right)^{2}}{\left(\hbar \omega_{1}+\hbar \omega_{2}\right)} .
$$

$\delta_{D}$ is associated with noncentrosymmetric systems and can be used to describe the 2PA spectra of molecule 1 while $\delta_{T}$ should provide a proper qualitative description of molecule 2 .
To study the reliability of the essential-state models to describe the resonance enhancement, we have compared the values for $\delta$ and ISRE obtained from the converged SOS approach (see Tables I and II) to those obtained from Eqs. (3) and (4). The relevant transition energies and dipole moments (as obtained from the INDO/MRD-CI calculations described above) are given in Table III; for molecule 1, only the properties of the one-photon state are given while for molecule 2 we also list the energy and transition dipole to the dominant 2PA active state in the spectral region of the 2PA maximum.

The comparison between the converged SOS results and the essential-state results is shown in Table IV. For both molecules, the ISRE is well reproduced by the essential-state models. However, the magnitude of the ISRE is somewhat underestimated, especially for molecule 1 . This is actually largely due to an overestimation of the degenerate 2PA cross section by the essential-state model. It turns out from a detailed analysis of the different channels contributing to the fully converged results that higher-lying one-photon allowed intermediate states result in mixed contributions which reduce $\delta$ (indeed, for the molecules investigated here, the linear absorption spectra in Fig. 2 show that there are additional strongly one photon allowed states around $4 \mathrm{eV}$ ). When the probe wavelength is tuned close to the first linear absorption, the $\delta_{D}$ [Eq. (3)] contribution to the overall 2PA into $|\mathbf{e}\rangle$ is much more strongly enhanced than the channels involving these higher-lying excited states. Therefore, the relative contribution of those channels decreases in the case of ISRE. This explains why the relative error in $\delta_{D}$ decreases for larger probe energies and the overall ISRE is smaller than for

\begin{tabular}{|c|c|c|c|c|c|c|c|}
\hline \multicolumn{4}{|c|}{ Molecule 1} & \multicolumn{4}{|c|}{ Molecule 2} \\
\hline$\hbar \omega_{1}$ & $\hbar \omega_{2}$ & $\delta_{D}$ & $\operatorname{ISRE}_{D}$ & $\hbar \omega_{1}$ & $\hbar \omega_{2}$ & $\delta_{T}$ & $\mathrm{ISRE}_{T}$ \\
\hline 1.72 & 1.72 & 135 & 1.00 & 2.03 & 2.03 & 99 & 1.00 \\
\hline 2.06 & 1.38 & 168 & 1.25 & 2.41 & 1.65 & 130 & 1.31 \\
\hline 2.41 & 1.03 & 225 & 1.67 & 2.68 & 1.38 & 179 & 1.80 \\
\hline 2.61 & 0.83 & 279 & 2.07 & 3.03 & 1.03 & 365 & 3.66 \\
\hline 2.75 & 0.69 & 335 & 2.49 & & & & \\
\hline
\end{tabular}
the converged results. These deviations are much smaller in

TABLE IV. Degenerate and nondegenerate 2PA cross sections in GM units (1 $\times 10^{-50} \mathrm{~cm}^{4} \mathrm{sec}$ photon ${ }^{-1}$ molecule ${ }^{-1}$ ) and ISRE for molecule 1 and molecule 2 as obtained from the essentialstate models given in Eqs. (3) and (4), respectively. $\hbar \omega_{1}$ and $\hbar \omega_{2}$ are given in $\mathrm{eV}$. 
molecule 2, since there the coupling via the higher-lying onephoton allowed states is weaker. ${ }^{45}$

Thus, the essential-state models provide a qualitative (and, in the case of molecule 2, also quantitative) description of ISRE, which allows a simple analysis of ISRE in terms of Eqs. (3) and (4). One finds a resonance enhancement for $\delta_{D}$ when either $\hbar \omega_{1}$ or $\hbar \omega_{2}$ approaches zero, which for 2PA into $|e\rangle$ requires $\hbar \omega_{2} \rightarrow E_{g e}$ or $\hbar \omega_{1} \rightarrow E_{g e}$. This is fully consistent with the experimental finding that the ND-2PA cross section strongly increases when the energy of the probe photon approaches the one-photon resonance. The resonances in $\delta_{T}$ also occur, when $\hbar \omega_{2} \rightarrow E_{g e}$ or $\hbar \omega_{1} \rightarrow E_{g e}$, rationalizing the increase in the cross section when the probe energy in the experiments approaches the energy of the intermediate onephoton state.

\section{SUMMARY AND CONCLUSIONS}

We have investigated the effect of resonance enhancement on the two-photon absorbing properties of both symmetric and asymmetric fluorene derivative. The use of ND2PA permitted us to control the photon energies involved in the 2PA process, allowing a quantitative study of the effect of ISRE in a single molecule. We were able to observe nearly five times enhancement in the value of the peak 2PA cross section for the symmetric compound. The asymmetric compound exhibited over threefold enhancement. Furthermore, 2PA into higher-lying excited states of the asymmetric compound revealed over 20 times enhancement of the nonlinearity.

Using a perturbative SOS expression including the first 300 excited states of the molecules under investigation, twophoton absorption spectra were calculated. The calculated spectra showed strong qualitative as well as quantitative agreement with the experimentally generated spectra for identical pump photon energies. A simplified three-level model for ND-2PA was also developed and provides insight into the mechanism of ISRE. These detailed studies of ND2PA reveal the potential to generate well over an order of magnitude enhancement of the nonlinearity as compared to D-2PA in the same molecule. This could provide great impetus for the development of applications which exploit ND2PA.

\section{ACKNOWLEDGMENTS}

E.W.V.S., D.J.H., and K.D.B. gratefully acknowledge the support of the National Science Foundation (Grant No. ECS0217932) and the Naval Air Warfare Center Joint Service Agile Program (Contract No. N00421-98-C-1327). K.D.B. would also like to acknowledge the donors of The Petroleum Research Fund of the American Chemical Society and the Research Corporation for partial support of this work. The work at Arizona/GeorgiaTech was supported, in part, by the STC program of the National Science Foundation under Agreement No. DMR-0120967, the AFOSR (Grant No. F49620-02-1-0358), and the IBM SUR program. P.P. acknowledges financial support by the Spezialforschungsbereich Elektroaktive Stoffe of the Austrian Fonds zur Förderung der Wissenschaftlichen Forschung.
${ }^{1}$ D. L. Pettit, S. S. H. Wang, K. R. Gee, and G. J. Augustine, Neuron 19, 465 (1997); E. A. Wachter, W. P. Partridge, W. G. Fisher, H. C. Dees, and M. G. Petersen, Proc. SPIE 3269, 68 (1998).

${ }^{2}$ W. Denk, J. H. Strickler, and W. W. Webb, Science 248, 73 (1990); R. H. Köhler, J. Cao, W. R. Zipfel, W. W. Webb, and M. R. Hansen, ibid. 276, 2039 (1997); M. J. Miller, S. H. Wei, I. Parker, and M. D. Cahalan, ibid. 296, 1869 (2002).

${ }^{3}$ D. A. Parthenopoulos and P. M. Rentzepis, Science 245, 843 (1989); J. H. Strickler and W. W. Webb, Opt. Lett. 16, 1780 (1991); B. H. Cumpston, S. P. Ananthavel, S. Barlow et al., Nature (London) 398, 51 (1999); S. Kawata, H.-B. Sun, T. Tanaka, and K. Takada, ibid. 512, 697 (2001); W. H. Zhou, S. M. Kuebler, K. L. Braun, T. Y. Yu, J. K. Cammack, C. K. Ober, J. W. Perry, and S. R. Marder, Science 296, 1106 (2002).

${ }^{4}$ L. W. Tutt and T. F. Boggess, Prog. Quantum Electron. 17, 299 (1993); A. A. Said, C. Wamsely, D. J. Hagan, E. W. van Stryland, B. A. Reinhardt, P. Roderer, and A. G. Dillard, Chem. Phys. Lett. 228, 646 (1994); J. D. Bhawalkar, G. S. He, and P. N. Prasad, Rep. Prog. Phys. 59, 1041 (1996); J. E. Ehrlich, X. L. Wu, Ys. L. Lee, Z. Y. Hu, H. Rockel, S. R. Marder, and J. W. Perry, Opt. Lett. 22, 1843 (1997).

${ }^{5}$ U. Gubler and C. Bosshard, Adv. Polym. Sci. 158, 123 (2002).

${ }^{6}$ O. Mongin, L. Porrès, L. Moreaux, J. Mertz, and M. Blanchard-Desce, Org. Lett. 4, 719 (2002); P. Cronstrand, Y. Luo, and H. Agren, Chem. Phys. Lett. 352, 262 (2002); B. Strehmel, A. M. Sarker, and H. Detert, ChemPhysChem 4, 249 (2003).

${ }^{7}$ E. Zojer, D. Beljonne, T. Kogej, H. Vogel, S. R. Marder, J. W. Perry, and J. L. Bredas, J. Chem. Phys. 116, 3646 (2002).

${ }^{8}$ M. Rumi, J. E. Ehrlich, A. A. Heikal et al., J. Am. Chem. Soc. 122, 9500 (2000).

${ }^{9}$ J. D. Bhawalkar, G. S. He, C.-K. Park, C. F. Zhao, G. Ruland, and P. N. Prasad, Opt. Commun. 124, 33 (1996); G. S. He, L. Yuan, N. Cheng, J. D. Bhawalkar, P. N. Prasad, L. L. Brott, S. J. Clarson, and B. A. Reinhardt, J. Opt. Soc. Am. B 14, 1079 (1997); G. S. He, L. Yuan, P. N. Prasad, A. Abotto, A. Facchetti, and G. A. Pagani, Opt. Commun. 140, 49 (1997); A. Abotto, L. Beverina, R. Bozio, S. Bradamante, C. Ferrante, G. A. Pagani, and R. Signorini, Adv. Mater. (Weinheim, Ger.) 12, 1963 (2000); Y. Luo, P. Norman, P. Macak, and H. Agren, J. Phys. Chem. 104, 4718 (2000); R. Zaleśny, W. Bartowiak, S. Styrcz, and J. Leszczynski, J. Phys. Chem. A 106, 4032 (2002); H. Lei, Z. L. Huang, H. Z. Wang, X. J. Tang, L. Z. Wu, G. Y. Zhou, D. Wang, and Y. B. Tian, Chem. Phys. Lett. 352, 240 (2002).

${ }^{10}$ H. Lee, S.-Y. An, and M. Cho, J. Phys. Chem. B 103, 4992 (1999); S.-J. Chung, K.-S. Kim, T.-Ch. Lin, G. S. He, J. Swiatkiewicz, and P. N. Prasad, ibid. 103, 10741 (1999); W.-H. Lee, H. Lee, J.-A. Kim, J.-H. Choi, M. Cho, S.-J. Jeon, and B. R. Cho, J. Am. Chem. Soc. 123, 10658 (2001); B. R. Cho, M. J. Piao, K. H. Son, S. H. Lee, S. J. Yoon, S.-J. Jeon, and M. Cho, Chem.-Eur. J. 8, 3907 (2002).

${ }^{11}$ R. R. Birge and B. M. Pierce, Int. J. Quantum Chem. 29, 639 (1986).

${ }^{12}$ S. K. Pati, T. J. Marks, and M. A. Ratner, J. Am. Chem. Soc. 123, 7287 (2001).

${ }^{13}$ T. Kogej, D. Beljonne, F. Meyers, J. W. Perry, S. R. Marder, and J. L. Bredas, Chem. Phys. Lett. 298, 1 (1998).

${ }^{14}$ M. Barzoukas and M. Blanchard-Desce, J. Chem. Phys. 113, 3951 (2000).

${ }^{15}$ M. Drobizhev, A. Karotki, M. Kruk, and A. Rebane, Chem. Phys. Lett. 355, 175 (2002).

${ }^{16}$ K. Kamada, K. Ohta, I. Yoichiro, and K. Kondo, Chem. Phys. Lett. 372, 386 (2003).

${ }^{17}$ R. Baltramiejunas, V. Gavryushin, G. Raciukaitis, and V. Kubertavicius, Fizika Tverdogo Tela 30, 1089 (1988).

${ }^{18}$ K. D. Belfield, K. J. Schafer, W. Mourad, and B. A. Reinhardt, J. Org. Chem. 65, 4475 (2000).

${ }^{19}$ J. R. Lakowicz, Principles of Fluorescence Microscopy (Kluwer Academic, New York, 1999).

${ }^{20}$ M. Fischer and J. Georges, Chem. Phys. Lett. 260, 115 (1996).

${ }^{21}$ K. D. Belfield, M. V. Bondar, O. V. Przhonska, K. J. Schafer, and W. Mourad, J. Lumin. 97, 141 (2002).

${ }^{22}$ K. D. Belfield, S. Andrasik, K. J. Schafer, O. Yavuz, J. M. Hales, and E. W. Van Stryland, Polym. Prepr. (Am. Chem. Soc. Div. Polym. Chem.) 84, $732(2001)$.

${ }^{23}$ C. Xu and W. W. Webb, J. Opt. Soc. Am. B 13, 481 (1996).

${ }^{24}$ S. M. Kennedy and F. E. Lytle, Anal. Chem. 58, 2643 (1986).

${ }^{25}$ R. A. Negres, J. M. Hales, A. Kobyakov, D. J. Hagan, and E. W. Van Stryland, IEEE J. Quantum Electron. 38, 1205 (2002).

${ }^{26}$ M. J. S. Dewar, E. G. Zoebisch, E. F. Healy, and J. J. P. Steward, J. Am. Chem. Soc. 107, 3902 (1985). 
${ }^{27}$ J. A. Pople, D. L. Beveridge, and P. A. Dobosh, J. Chem. Phys. 47, 2026 (1967); J. Ridley and M. Zerner, Theor. Chim. Acta 32, 111 (1973).

${ }^{28}$ R. J. Buenker and S. D. Peyerimhoff, Theor. Chim. Acta 35, 33 (1974).

${ }^{29}$ As reference determinants in the MRD-CI calculations we chose the determinants most strongly contributing to a single and double CI description of the dominant one- and two-photon states. These are (i) the SCF determinant; (ii) a determinant in which an electron has been promoted from the HOMO to the LUMO; (iii) one in which an electron has been excited from the HOMO-1 to the LUMO; (iv) the doubly excited determinant in which both electrons are excited from the HOMO to the LUMO; and (v) for molecule 1 the HOMO to LUMO+1 and for molecule 2 the $\mathrm{HOMO}$ to LUMO +5 determinants. The $\mathrm{CI}$ active space for the single excitations included the 24 highest occupied and 24 lowest unoccupied orbitals in molecule 1 ; for molecule 2 , it was increased to $28 \times 28$ orbitals due to the larger number of $\pi$ electrons. For the higher excitations, in all cases five occupied and five unoccupied orbitals have been considered.

${ }^{30}$ N. Mataga and K. Nishimoto, Z. Phys. Chem. (Munich) 13, 140 (1957).

${ }^{31}$ The WLC pump-probe method, as described in Ref. 25, actually extracts the nondegenerate $2 \mathrm{PA}$ coefficient $\beta_{\mathrm{ND}}$ from the experimental data. In order to generate the nondegenerate $2 \mathrm{PA}$ cross section $\delta_{\mathrm{ND}}$ the expression for $\beta_{\mathrm{ND}}$ (Ref. 32) can be substituted into Eq. (1) using the well-known relationship between $\chi^{(3)}$ and $\gamma$ (Ref. 39),

$\delta_{\mathrm{ND}}=\frac{\hbar \beta_{\mathrm{ND}}}{N}\left(\frac{2 \omega_{1} \omega_{2}}{\omega_{1}+\omega_{2}}\right)$,

where $N$ is the number density of molecules in the system. This reduces to the well-known relationship between degenerate $\delta$ and $\beta$ when $\omega_{1}=\omega_{2}$.

${ }^{32}$ R. L. Sutherland, Handbook of Nonlinear Optics (Dekker, New York, 1996).
${ }^{33}$ B. E. Scharf and Y. B. Band, Chem. Phys. Lett. 144, 165 (1988).

${ }^{34}$ A. Painelli, L. D. Freo, and F. Terenziani, Chem. Phys. Lett. 346, 470 (2001).

${ }^{35}$ S. J. K. Pond, M. Rumi, M. D. Levin, T. C. Parker, D. Beljonne, M. W. Day, J. L. Brédas, S. R. Marder, and J. W. Perry, J. Phys. Chem. 106, 11470 (2002)

${ }^{36}$ B. J. Orr and J. F. Ward, Mol. Phys. 20, 513 (1971).

${ }^{37}$ The expression for $\delta_{\mathrm{ND}}$ can be derived by following the photon flux approach detailed in Ref. 38 for a two-beam interaction. The necessary relationship between $\chi^{(3)}$ and $\gamma$ can be found in Ref. 39 .

${ }^{38}$ B. Dick, R. M. Hochstrasser, and H. P. Trommsdorff, Nonlinear Optical Properties of Organic Molecules and Crystals (Academic, Orlando, 1987).

${ }^{39}$ R. W. Boyd, Nonlinear Optics (Academic, San Diego, 1992).

${ }^{40}$ C. W. Dirk, L. Cheng, and M. G. Kuzyk, Int. J. Quantum Chem. 43, 27 (1992).

${ }^{41}$ R. R. Birge and B. M. Pierce, J. Chem. Phys. 70, 165 (1979).

${ }^{42}$ S. Mazumdar, D. Duo, and S. N. Dixit, Synth. Met. 55-57, 3881 (1993); S. Mazumdar and F. Guo, J. Chem. Phys. 100, 1665 (1994).

${ }^{43}$ P. Pacher, Diploma thesis, Graz University of Technology, 2003.

${ }^{44}$ The factor $1 / 5$ is obtained for an isotropic medium assuming that the transition dipoles and state-dipole changes are parallel.

${ }^{45}$ The quantum-chemical calculations reveal that the peak at $4.06 \mathrm{eV}$ is, in fact, a superposition of $2 \mathrm{PA}$ into several excited states. Only the strongest of those states (the one used for the analysis in Table IV) displays any significant coupling to the higher-lying intermediate states. Therefore, for this 2PA-active state, a small underestimation of ISRE by the essentialstate model can be expected while the contributions of the other 2PAactive states to the ISRE are better described by the essential-state model. 